\title{
Case report: Calcifying fibrous tumor presenting as an asymptomatic pelvic mass
}

\author{
Angela A Giardino ${ }^{1,4}$, Nikhil H Ramaiya ${ }^{1,4}$, Atul B Shinagare ${ }^{1,4}$, Jyothi P Jagannathan ${ }^{1,4}$, \\ Matthew D Stachler 2,4, Chandrajit P Raut ${ }^{3,4}$
}

'Department of Imaging, Dana-Farber Cancer Institute, 450 Brookline Ave, Boston, MA 02115 and Departments of Radiology and 'Pathology, Brigham and Women's Hospital, 75 Francis Street, Boston, MA 02115, 'ंDepartment of Surgery, Brigham and Women's Hospital, 75 Francis Street, Boston, MA 02115, and Center for Sarcoma and Bone Oncology, Dana-Farber Cancer Institute, 450 Brookline Ave, Boston, MA 02115, ${ }^{4}$ Harvard Medical School, 25 Shattuck Street, Boston, MA 02115 USA

Correspondence: Atul B Shinagare, Department of Imaging, Dana-Farber Cancer Institute, 450 Brookline Ave, Boston, MA 02115 USA. E-mail: ashinagare@partners.org

\section{Abstract \\ Calcifying fibrous tumor (CFT) is a rare benign mesenchymal tumor most commonly found in the soft tissues of the extremities and pleura. It is characterized by hyalinized collagenous fibrous tissue, with bland spindle cells, psammomatous or dystrophic calcifications, and focal lymphoplasmacytic infiltrate. CFT of the gastrointestinal tract is exceedingly uncommon. We report a case of CFT arising from the small intestine and associated mesentery; this case was identified incidentally in an otherwise healthy 45-year-old man.}

Key words: Calcifying fibrous tumor; mesentery; small intestine

\section{Introduction}

Calcifying fibrous tumor (CFT) is a rare, usually solitary, benign tumor of uncertain etiology. We report an incidentally detected case of CFT arising from the small intestine and associated mesentery.

\section{Case Report}

A 45-year-old man with no significant past medical history presented to a local emergency room with severe left groin pain. His evaluation included a CT scan of the abdomen and pelvis, which showed a $5 \mathrm{~cm}$ densely calcified right pelvic mass [Figure 1]. The mass was considered an incidental

\begin{tabular}{|l|l|}
\hline \multicolumn{2}{|c|}{ Access this article online } \\
\hline Quick Response Code: & \\
\hline & Website: \\
\hline & www.ijri.org \\
\cline { 2 - 3 } & DOI: \\
\hline
\end{tabular}

finding and unrelated to his presenting symptoms. The remainder of his workup was negative. No biopsy of the mass was performed at that time.

He was subsequently referred to our Center for Sarcoma and Bone Oncology with a presumed diagnosis of soft tissue sarcoma. Upon initial consultation, the treatment options discussed included obtaining a preoperative, diagnostic, image-guided core needle biopsy or proceeding directly to surgery. When it was explained to him that this mass required resection in any case and that the findings of a preoperative biopsy would not change treatment plans, the patient elected to proceed with surgery. At laparotomy, he was found to have a hard mass involving the distal small intestine. The mass was adherent to the intestinal serosa [Figure 2]. An extension of the mesentery appeared to represent the vascular supply to the mass. A small bowel resection with an en bloc resection of the involved portion of the mesentery was performed. A primary enteroenterostomy was constructed.

Histopathologic evaluation was consistent with a CFT. The tumor exhibited a relatively hypocellular spindle cell 


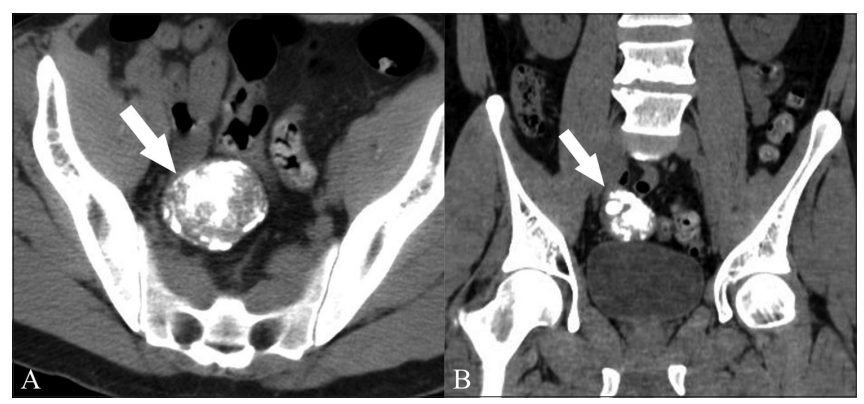

Figure $1(A, B)$ : Axial $(A)$ and coronal $(B)$ noncontrast $C T$ scans show a $5 \mathrm{~cm}$, densely calcified pelvic mass (arrow) and its intimate relationship with the adjacent small bowel loops

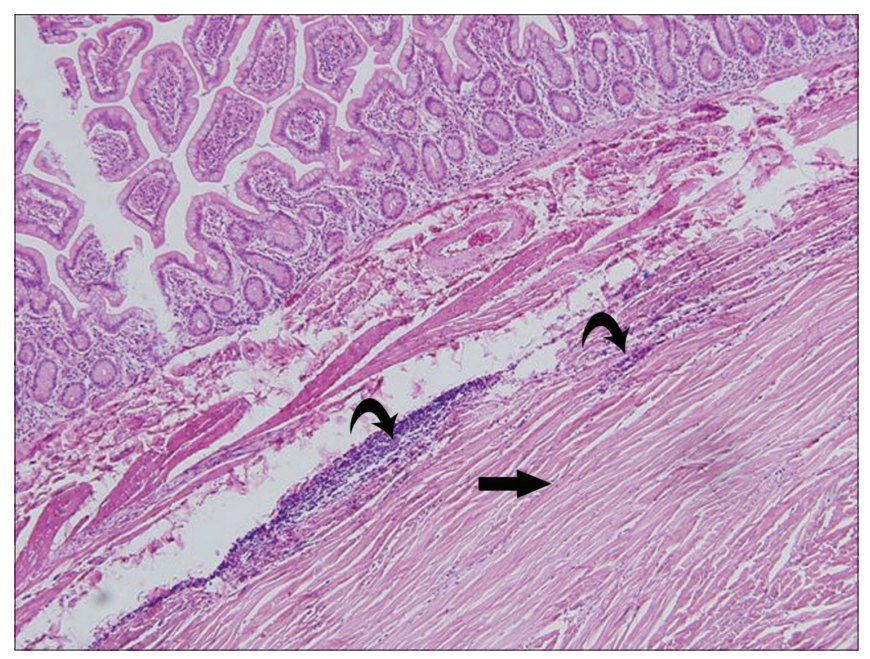

Figure 3: Low-power (10x) photomicrograph stained with hematoxylin and eosin shows relatively hypocellular spindle cell proliferation (bottom right corner), with dense collagen fibers (arrow) and scattered to loosely aggregated lymphocytes and plasma cells (curved arrows)

proliferation, with dense collagen fibers and scattered to loosely aggregated lymphocytes and plasma cells [Figure 3]. The mass was well circumscribed, with smooth borders. Intestinal and mesenteric resection margins were negative.

Based on this diagnosis, no further treatment was needed or recommended. The patient was followed up after 1 year. Repeat CT scan (not shown) showed no evidence of recurrence.

\section{Discussion}

CFT is a rare, usually solitary, benign tumor of uncertain etiology. The most common sites are the soft tissues of the extremities, pleura, or peritoneum. ${ }^{[1-3]}$ Involvement of the gastrointestinal (GI) tract is rare. About 30 cases of abdominal CFTs have been reported to date. ${ }^{[3]}$ For abdominal CFTs the mean patient age is 34 years compared with 16 years for patients with CFTs occurring in the superficial soft tissues. ${ }^{[3]}$ CFTs have characteristic histologic features

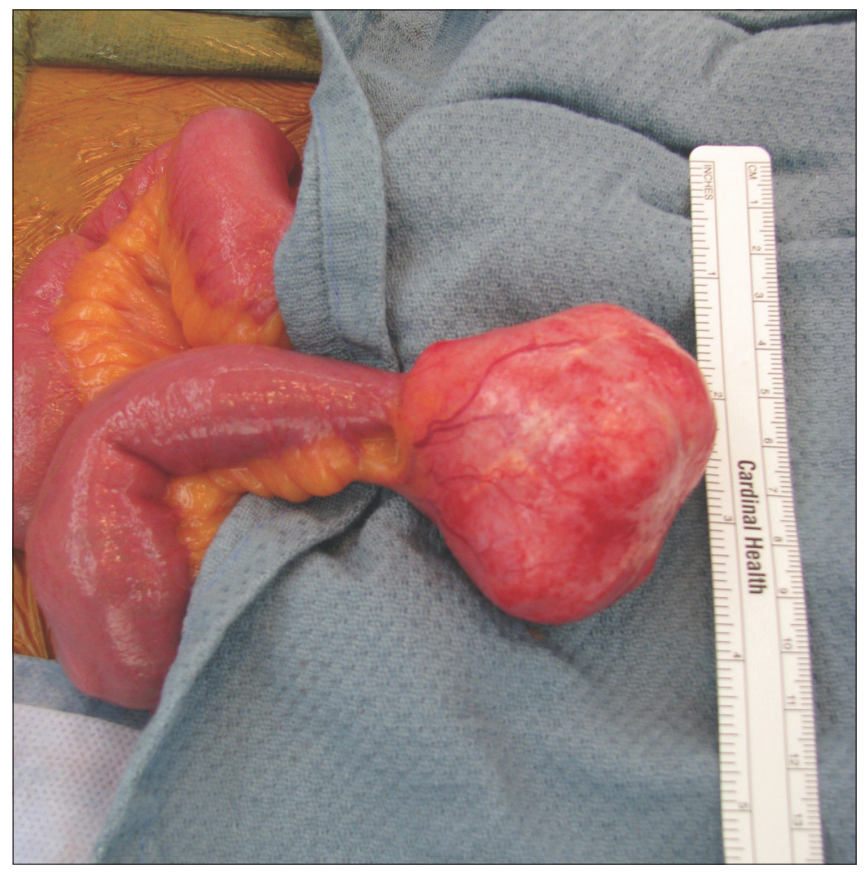

Figure 2: Intraoperative photograph shows a pedunculated mass on the small bowel mesentery

of a well-circumscribed mass composed of hyalinized, hypocellular, lamellar collagen; bland spindle cells; chronic inflammatory cell infiltrates; and psammomatous or dystrophic calcifications. ${ }^{[2,3]}$ Thought to represent a reactive process resulting from abnormal tissue healing, the lesions were previously called calcifying fibrous pseudotumor. However, due to a local recurrence rate of approximately $10 \%$, these lesions were renamed CFTs in the current World Health Organization classification. ${ }^{[1,4]}$

The most important differential diagnosis of CFT of the GI tract includes other spindle cell tumors, such as desmoid tumor and gastrointestinal stromal tumor (GIST). ${ }^{[2]}$ Desmoid tumors have a variable CT appearance depending on fibroblastic proliferation, fibrosis, collagen content, and vascularity ${ }^{[5]}$ On CT scan, they usually appear as well circumscribed, homogeneous masses that may enhance with intravenous contrast. ${ }^{[5]}$ Most intraabdominal desmoids arise in the small bowel mesentery. ${ }^{[5]}$ GISTs may occur along any part of the alimentary tract that contains smooth muscle, with stomach and small bowel being the most common locations ${ }^{[6]}$ On CT scan, GISTs are usually enhancing, well-circumscribed solid masses. ${ }^{[6]}$ With increasing size there may be central necrosis, cavitation, and heterogeneous enhancement. ${ }^{[6]}$ GISTs have a submucosal origin with exophytic growth and displace rather than invade surrounding structures as they enlarge. ${ }^{[7]}$ Calcification is rare, however, a GIST of the sigmoid colon with a large calcified component has been reported. ${ }^{[6]}$ Lymph node involvement would not be expected in CTF, desmoid, or GIST. 
Other radiologic differential considerations for a calcified pelvic mass include calcified lymph node, carcinoid, inflammatory myofibroblastic tumor (IMT), sclerosing mesenteritis, and extraskeletal osteosarcoma. Ovarian cystadenoma/carcinoma should also be considered in females.

A calcified lymph node may be the result of prior granulomatous disease or a treated lymphoma ${ }^{[8]}$ Correlation with the clinical history is also important. While small intestine carcinoids may extend into the mesentery, calcification in the mass, particularly dense calcification, is unusual. ${ }^{[9]}$ IMT and sclerosing mesenteritis are included in a group of benign fibrous tumors / tumor-like lesions of the mesentery, linked histologically by the presence of fibroblasts or fibrosis and anatomically by location within the mesentery. ${ }^{[10]}$ IMT is a benign, chronic inflammatory disorder of unknown etiology that may manifest as a solid mesenteric mass. ${ }^{[10]}$ The enhancement pattern is variable and calcification may be present. ${ }^{[10]}$ Sclerosing mesenteritis is a rare idiopathic disorder characterized by mesenteric masses composed of chronic, nonspecific inflammation. ${ }^{[10]}$ On CT scan, sclerosing mesenteritis may manifest as a well-defined mesenteric mass with calcification. ${ }^{[10]}$

A rare case of extraskeletal osteosarcoma of the mesentery presenting as a large calcified mass in the pelvic cavity has been reported..$^{[11]} \mathrm{CT}$ scan findings are nonspecific and overlap with multiple other entities, and therefore warrant biopsy/excision. Accurate histologic diagnosis requires specific immunohistochemisties, including ALK1. Histologic distinction is imperative for the correct treatment and proper prognosis.

Resection of CFT is usually curative but because of the potential for local recurrences, patients should undergo periodic follow-up. There are no guidelines for specific screening or follow-up radiologic imaging. ${ }^{[2]}$

In summary, we present a patient with a rare case of CFT of the GI tract discovered incidentally at imaging and treated with surgical resection. The patient is recurrence free at 1 year. In the absence of any distinctive imaging characteristics to distinguish CFTs from other rare intraabdominal soft tissue neoplasms, biopsy and/or resection is recommended in all cases.

\section{References}

1. Chen K. Familial peritoneal multifocal calcifying fibrous tumor. Am J Clin Pathol 2003;119:811-5.

2. Emanuel P, Qin L, Harpaz N. Calcifying fibrous tumor small intestine. Ann Diagn Pathol 2008;12:138-41.

3. Jo BJ, Yoon SW, Ahn HJ, Kwon SW. Imaging findings of calcifying fibrous tumour of the liver. Br Radiol 2011;84:e31-4.

4. Agaimy A, Bihl MP, Tornillo L, Wünsch PH, Hartmann A, Michal M. Calcifying fibrous tumor of the stomach: Clinicopathologic and molecular study of seven cases with literature review and reappraisal of histogenesis. Am J Surg Pathol 2010; 34:271-8.

5. Faria SC, Iyer RB, Rashid A, Ellis L, Whitman GJ. Desmoid tumor of the small bowel and the mesentery. AJR Am J Roentgenol 2004;183:118.

6. Rana R, Nikolaidis P, Miller F.Calcified gastrointestinal stromal tumor of the sigmoid colon mimicking inspissated barium on CT. AJR Am J Roentgenol 2006;187:W322-3.

7. Lau S, Tam KF, Kam CK, Lui CY, Siu CW, Lam HS, et al. Imaging of gastrointestinal stromal tumour (GIST). Clin Radiol 2004;59:487-98.

8. Lucey BC, Stuhlfaut JW, Soto JA. Mesenteric lymph nodes seen at imaging: Causes and significance. Radiographics 2005;25:351-65.

9. Pantongrag-Brown L, Buetow PC, Carr NJ, Lichtenstein JE, Buck JL. Calcification and fibrosis in mesenteric carcinoid tumor: CT findings and pathologic correlation. Am J Roentgenol 1995;164: 387-91.

10. Levy AD, Rimola J, Mehrotra AK, Sobin LH. From the archives of the AFIP: Benign fibrous tumors and tumorlike lesions of the mesentery: Radiologic-pathologic correlation. Radiographics 2006;26:245-64.

11. Choudur HN, Munk PL, Nielson TO, Ryan AG. Primary mesenteric extraskeletal osteosarcoma in the pelvic cavity. Skeletal Radiol 2005;34:649-52.

Cite this article as: Giardino $\mathrm{AA}$, Ramaiya $\mathrm{NH}$, Shinagare $\mathrm{AB}$, Jagannathan JP, Stachler MD, Raut CP. Case report: Calcifying fibrous tumor presenting as an asymptomatic pelvic mass. Indian J Radiol Imaging 2011;21:306-8.

Source of Support: Nil, Conflict of Interest: None declared. 